\title{
Le rendez-vous des \\ immunologistes européens à Paris
}

Nathalie Bonnefoy-Bérard,

Nathalie Auphan-Anezin,

Catherine Sautès-Fridman
Le $16^{\mathrm{e}}$ congrès européen d'immunologie s'est tenu du 6 au 9 septembre 2006 au Palais des Congrès de Paris (France). Pour la première fois, et sous l'impulsion de la Société française d'immunologie (SFI), la plupart des sociétés européennes d'immunologie ont accepté d'annuler leur propre congrès annuel afin de donner naissance à la première réunion conjointe des sociétés nationales européennes d'immunologie. Cette nouvelle organisation, en permettant le rassemblement de plus de 5000 scientifiques, a contribué au succès de ce congrès, d'autant que plus de 80 pays étaient représentés, donnant une envergure réellement mondiale à cette réunion. Ce succès est également le fruit d'une qualité et d'une diversité excellentes du programme scientifique, ainsi que de la large place réservée aux étudiants en thèse (1 400 participants). Le programme scientifique était construit autour de quatre grands thèmes: immunité innée ou naturelle, immunité acquise ou adaptative, immunopathologie et, enfin, immunointervention. Ces grands axes ont ainsi permis de couvrir la physiologie et la pathologie du système immunitaire.

\section{Des sessions remarquables}

\section{Infections virales et vaccination}

Parmi les sujets majeurs abordés, cette session a permis de faire le point sur la vaccination antivirale dans le contexte de pathologies telles que les infections chroniques par les virus de l'immunodéficience humaine (VIH) ou de l'hépatite C (VHC).

Andrew McMichael (Oxford, Royaume-Uni) et Brigitte Autran (Paris, France) ont insisté sur la nécessité de mettre au point un vaccin prophylactique ou thérapeutique contre le VIH. II a été rappelé que le principal obstacle au développement d'un vaccin prophylactique est la très grande difficulté à obtenir des anticorps neutralisants efficaces, en raison de problèmes liés

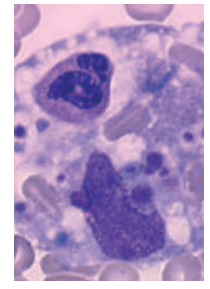

à la conformation des protéines virales, au fait que d'éventuels sites de neutralisation puissent être masqués et, enfin, à la très grande variabilité virale, notamment au niveau de l'enveloppe. Pour limiter l'intensité de l'infection et la progression de la maladie, les stratégies actuelles s'orientent davantage vers une induction des lymphocytes T CD8 et CD4 effecteurs que vers l'induction des anticorps. Des stratégies de type primeboost, combinant un inducteur d'une réponse mémoire ADN et des vecteurs poxvirus ou adénovirus recombinants, semblent pouvoir amplifier une réponse lymphocytaire $T$ spécifique. Des essais cliniques de phases I et II utilisant de telles stratégies confirment l'induction d'une réponse immune: les premiers résultats des études utilisant un poxvirus aviaire (canarypox) recombinant pour plusieurs gènes du $\mathrm{VIH}$, seul ou en association avec des particules virales inactivées, notamment, semblent encourageants. Par ailleurs, une prolongation significative de l'interruption de la chimiothérapie (de type HAART, highly active antiretroviral therapy) et une diminution de la réplication virale ont pu être obtenues lorsque cette stratégie est utilisée uniquement en phase chronique, et non en phase d'infection primaire. Les facteurs influençant ces résultats pourraient tenir à l'intensité des réponses immunes pré-existantes, supérieures en phase chronique. 


\section{Contrôle local des tumeurs}

Au cours de cette session, Robert Schreiber (Saint-Louis, États-Unis) a présenté les résultats de son équipe sur l'analyse des relations entre hôte et tumeur. Le cancer se développe selon trois phases : une phase d'élimination, pendant laquelle l'hôte se défend contre la tumeur, une phase d'équilibre, où la tumeur est modifiée par le système immunitaire, et une phase d'échappement, au cours de laquelle la tumeur modifiée échappe au système immunitaire et se développe. L'induction des réponses immunitaires nécessite le contact intime entre les différentes sous-populations des cellules du système immunitaire.

Dans ce contexte, Marie-Caroline Dieu-Nosjean (Paris, France) a présenté des images montrant l'existence, dans les poumons de patients atteints de cancers du poumon, de structures lymphoïdes ectopiques comprenant des cellules dendritiques, des lymphocytes $T$ et des lymphocytes B. Cette présence, que l'on n'observe pas dans les poumons de sujets sains adultes, traduit l'activation d'une réponse immunitaire locale au contact de la tumeur, celle-ci étant infiltrée par des vaisseaux sanguins et lymphatiques.

De son côté, Kari Alitalo (Helsinki, Finlande) a démontré l'importance du couple VEGF-C/VEGFR-3 (vascular endothelium growth factor et -receptor) au cours de l'embryogenèse et de la progression tumorale: de fait, la surexpression du VEGF-C favorise le développement de nouveaux vaisseaux lymphatiques intratumoraux ainsi que l'apparition de métastases ganglionnaires, des effets inhibés par le VEGFR-3-Ig (forme soluble du domaine extracellulaire du VEGFR-3). Ces résultats suggèrent l'existence d'une communication dynamique entre les cellules tumorales et l'endothélium lymphatique au cours du processus métastatique.

\section{Dernières nouveautés}

Deux sessions consacrées aux late breaking news ont permis au comité scientifique de mettre en évidence de récents points forts des recherches en immunologie.

Jérôme Galon (Paris, France) a montré, au cours d'une étude menée à grande échelle sur des tumeurs provenant de patients atteints d'un cancer colorectal, que le niveau de lymphocytes T CD8 effecteurs mémoires infiltrant le tissu tumoral est inversement corrélé au caractère invasif de la tumeur, et qu'il s'agit d'un facteur de bon pronostic. Par ailleurs, le devenir des patients, en termes de survie et de développement de métastases, n'est corrélé ni au contexte inflammatoire (interleukine-18, IL-18 ; tumor necrosis factor $\alpha$, TNF $\alpha$ ), ni au contexte suppresseur (IL-10, transforming growth factor $\beta$, TGF $\beta$ ).

Un lien magistral entre immunologie fondamentale et immunologie clinique a été démontré par Stefan Feske (Boston, États-Unis), qui a rapporté la caractérisation fonctionnelle d'une sous-unité des canaux calciques de type CRAC (calcium-release-activated calcium). La mutation de cette protéine abroge l'influx de calcium intracellulaire et, par conséquent, l'activation du facteur de transcription NF-AT (nuclear factor of activated T cells) impliqué dans le contrôle de l'expression génique d'un grand nombre de médiateurs de la réponse immunitaire. Elle est également à l'origine d'un type de syndrome de déficit immunitaire sévère combiné (SCID, severe combined immunodeficiency) caractérisé par un défaut d'activation des lymphocytes $T$, et donc par une hypersensibilité aux infections virales et fongiques.

\section{Le point sur... les lymphocytes T régulateurs}

Ce congrès européen a également été l'occasion de faire le point sur les derniers travaux de caractérisation des lymphocytes T régulateurs, destinés notamment à développer de nouvelles approches d'immunothérapie. Ainsi, le groupe de Jeffrey Bluestone (San Francisco, États-Unis) utilise des anticorps monoclonaux anti-CD3 afin d'induire l'expansion de lymphocytes $T$ régulateurs chez des patients souffrant d'un diabète de type 1. De fait, chez les souris NOD (non obese diabetic), qui développent spontanément un diabète de type 1 , l'injection d'anti-CD3 induit en 6 jours la disparition des lymphocytes T effecteurs infiltrant et détruisant les îlots pancréatiques. La rémission, qui peut être observée pendant 20 semaines, est liée à l'expansion des lymphocytes T régulateurs chez les souris traitées. Une approche d'expansion in vitro de ces cellules régulatrices a été mise au point, et adaptée afin d'augmenter leur fonction suppressive, et donc leur activité après transfert chez l'animal. Chez l'homme, l'injection d'anticorps anti-CD3 chez des patients recevant une greffe de rein induit une tolérance au greffon; dans ce cas, l'effet du traitement pourrait être lié à une augmentation de l'expression du récepteur PD-1 (programmed death-1), une protéine inhibant l'activation des cellules T effectrices.

\section{Remises de prix}

Le prix Schering-Plough, attribué par la fédération des sociétés européennes d'immunologie ( $\varepsilon F I S)$, a été décerné au Pr Alberto Mantovani, directeur scientifique du Instituto Clinico Humanitas et président de la fondation de recherche Humanitas à Milan. Ce prix récompense ses travaux démontrant le rôle clé joué par les chimiokines dans l'infiltration des tumeurs par les leucocytes et dans le trafic des cellules dendritiques. Ce prix a également couronné ses travaux sur les decoy receptors, ces récepteurs, dépourvus de région intracytoplasmique, impliqués dans la régulation de réponses inflammatoires par séquestration du ligand.

Le prix Jacques Oudin est attribué chaque année par la Société française d'immunologie et le Laboratoire français du fractionnement et des biotechnologies (LFB) pour des recherches effectuées en immunologie clinique. II a été attribué à Frédéric Rieux-Laucat (Paris, France) pour l'ensemble de ses travaux sur la mise en évidence des défauts de la voie de signalisation Fas dans l'apparition du syndrome lymphoprolifératif auto-immun, ainsi que pour ses travaux, plus récents, décrivant une mutation homozygote germinale du gène codant pour la sous-unité $C D 3 \zeta$ du complexe TCR ( $T$-cell receptor)$\mathrm{CD} 3$ chez un patient présentant une immunodéficience $T$ sévère.

Enfin, le prix Avery Landsteiner, décerné chaque année également par la société allemande d'immunologie pour honorer la carrière d'un immunologiste de renom, a récompensé Philippa Marrack (Denver, États-Unis) pour sa contribution essentielle à la découverte de la structure et de la fonction du récepteur des lymphocytes T. $\diamond$ The «rendez-vous » of the European immunologists in Paris 